\title{
Isolation and Analysis of Mycobacteriophage
}

Authors: Jennifer A. Jung and Aaron Powell

Faculty Mentors: Katherine E. Deming, Robert C. Benjamin, and Lee E. Hughes Department of Biological Sciences, College of Arts and Sciences

College and Department Affiliation: Department of Biological Sciences, College of Arts and Sciences 


\section{Bios:}

Jennifer Allison Jung hales from Irving, Texas. She is a junior majoring in biological sciences at the University of North Texas and is a member of the Honors College.

Aaron Lamon Powell is a freshman at the University of North Texas. He is majoring in biological sciences. 


\begin{abstract}
:
Mycobacteriophage are viruses that attack and infect mycobacterial hosts, such as Mycobacterium smegmatis. Bacteriophage are the most abundant and varying biological entities in the biosphere. The mycobacteriophage CharlieBrwn was isolated from an enriched soil sample collected from a home in DeSoto, TX using M. smegmatis as a host bacteria. CharlieBrwn was isolated and purified using standard procedures such as spot tests, streaking, and assays, and was then characterized by plaque morphology, electron microscopy, restriction digestion and gel electrophoresis. The plaques were cloudy, comet-shaped and about $2 \mathrm{~mm}$ in diameter. After further characterization, CharlieBrwn is suggested to be classified in the mycobacteriophage subcluster A2. Additional research on this and related bacteriophages could prove to be very beneficial to the development of phage therapy strategies to treat important diseases such as leprosy and tuberculosis.
\end{abstract}




\section{Introduction}

Bacteriophage are viruses that use bacterial cells as hosts. Mycobacteriophage are bacteriophage that use specifically bacteria of the genus Mycobacterium as their host. While the research on bacteriophage (phage) is not a particularly new field, recent developments and findings have high potential to aide biologists in many ways. For example, some phages easily manipulate the genetic makeup of their bacterial host by inserting their DNA and integrating it with the host's DNA (1). Because of this simple, yet resourceful trait, phages have been used as tools for either genetic manipulation of or the identification of certain pathogenic bacteria. For example, mycobacteriophages, specifically, could be used to diagnose Mycobacterium tuberculosis (2). Or another possibility could be the progression in the development of phage therapy, where phages are used as tools to cure or help fight off bacterial diseases (3). Further research on phages and their genomes would ensure the future of biomedical success in the control of bacterial infections.

\section{Materials and Methods}

CharlieBrwn was isolated and analyzed using protocols and procedures set forth in the Howard Hughes Medical Institute (HHMI) National Genomics Research Initiative (NGRI) SEAPHAGES Laboratory Manual (4). The lab manual is separated into three sections: isolation, purification, and characterization. To begin isolation, we enriched the soil sample by following the "Harvesting and Preparing the Enriched Sample" procedure using M. smegmatis as the host bacteria.

Purification began with a spot test using the procedure "Spotting Putative Plaques and Buffer Control onto a Labeled Plate". After a successful spot plate, two different plaques moved forward to streaking. The purpose of streaking is to ensure that the phage that moves forward is 
the purest of the pure. The "Plaque Streak Protocol" made this possible by streaking three separate times and getting the same plaque morphology each time. In "Plaque Streak Protocol" a negative control was not streaked. Purification continued with a phage-titer assay, another spot test, 10-fold dilutions, empirical assays, and large plate infections. Many procedures were repeated due to a lack of results or a contamination. The following procedures were repeated multiple times: 10-fold serial dilutions (x2), Performing a Spot Test on Phage Lysate (x3), and Performing the Empirical Assay (x2). In "Performing the Large-Plate Infection" procedure, the following changes were made: we did not use a large volume of phage, M. smegmatis, or top agar (TA); we plated as "normal" (i.e. $5 \mathrm{~mL}$ of M. smegmatis, infect with phage, and add TA -each done 10 times). In "Titering Your Phage" procedure we did 10-fold dilutions to $10^{-10}$. While purifying the phage, multiple calculations were made for a medium titer lysate (MTL) and a high titer lysate (HTL). A MTL is a phage lysate concentration that is simply used to calculate the HTL, the amount of phage needed to create a web plate. A web plate results when the amount of plaques find the perfect middle ground between completely isolated plaques and an obliterated plate. An example is show below in Figure 1.

Characterization was done through restriction digest, gel electrophoresis, and electron microscopy. In "Isolate and Purify Phage Genomic DNA" procedure the following was changed: skipped step C - did not re-suspend pellet; used $0.5 \mathrm{~mL}$ of resin instead of $1.25 \mathrm{~mL}$ of water resin in step F; used $50 \mu \mathrm{L}$ of nuclease free water instead of TE in step F6; used only $1 \mathrm{~mL}$ of $80 \%$ isopropanol; and centrifuged at 13,000 rpm for 5 minutes the first time and 1 minute the second time. The NanoDrop equipment was used to quantify the DNA in CharlieBrwn's HTL. In the "Restrict and Analyze Phage Genomic DNA" procedure, a loading dye was extra glycerol was used because more than $7 \mu \mathrm{L}$ of DNA was mixed in with the restriction enzymes. The extra 
glycerol ensured that the DNA would sink to the bottom of the well in the gel. Once the gels were finished running and were photographed, computer software ImageJ and a semi-log graph were used to estimate genome size.

For electron microscopy prep, a copper grid was set in $30 \mu \mathrm{L}$ of CharlieBrwn's HTL for 2 minutes (to allow the phage to attach to the grid) and in acid dye for 30 seconds (to ensure contrast) as was called for in the protocol.

\section{Results}

During the isolation and purification of the CharlieBrwn, multiple pictures were taken of the phage and its plaques. It was found that the plaques often had comet tails, and was about 2 $\mathrm{mm}$ in diameter and $5 \mathrm{~mm}$ in length. The plaques were also very cloudy. Pictures of CharlieBrwn are shown below in Figure 2. Pictures of the comet tails are not available. CharlieBrwn's HTL calculated to have $1 \times 10^{10}$ plaque forming units $(\mathrm{pfu}) / \mathrm{mL}$.

Before moving on to restriction digests, the concentration of DNA in CharlieBrwn's HTL was determined to be $34.0 \mathrm{ng} / \mu \mathrm{L}$ using the NanoDrop. This gave a total DNA yield of $2.32 \mu \mathrm{g}$. However, it was enough to move forward with the restriction digests and gel electrophoresis, provided the heavier (extra glycerol) loading dye was used. CharlieBrwn's genome size estimated to be about 43,200 bp by preparing a standard curve using a DNA molecular weight ladder. The gel is shown in Figure 3 and the genome size calculation is shown in Table 1 and Figure 4. From the electron micrograph, CharlieBrwn's head subunit is estimated to be $60.0 \mathrm{~nm}$ in diameter and its tail subunit is estimated to be $133 \mathrm{~nm}$ in length. The results of the electron microscopy is shown below in Figure 5.

\section{Discussion}


Bacteriophages generally infect a host by injecting their DNA into a bacterium, forcing the host to produce more of the phage by hijacking or taking over the host's transcriptional and translational machinery, and then lysing the host. Phages can either be lytic or lysogenic. Lytic phages lyse all infected hosts, creating a very clear plaque; while lysogenic phages allow bacteria to multiply with the phage DNA, which creates a cloudier, opaque plaque. Because of the cloudy plaque morphology, CharlieBrwn is likely a lysogenic phage (5). Based upon the virtual digest, CharlieBrwn seems to belong in sub cluster A2. A comparison of the virtual digestions of CharlieBrwn and Pukovnik shows both to be cleaved extensively by BamHI and ClaI while both show few to no cuts by EcoRI and HindIII. The similarity of the digestions and the electron micrographs of CharlieBrwn and the A2 phage are shown below in Figures 6 and 7. The virtual digest shown is of the phage Pukovnik, a phage in PhagesDB (6). The electron micrographs shown are of phages L5, Jsquared, and Pukovnik, all A2 phages in PhagesDB. Most A2 phages are lysogenic, further evidence that CharlieBrwn may be an A2 phage.

The average genome size for an A2 cluster is 52,671 bp. CharlieBrwn's estimated genome size of 43,200 bp is slightly smaller than the average genome size in subcluster A2. Since estimate genome size of CharlieBrwn was determined by a standard curve on a logarithmic scale using the DNA molecular weight ladder, any bands outside of the curve are very difficult to estimate. Or it could be that the estimation is wrong from the low concentration of DNA in the HTL because the less DNA there is, the higher error possibility. Or CharlieBrwn could simply be a unique phage in subcluster A2. 


\section{References}

1) Petty, N.K., T.J. Evans, P.C. Fineran, and G.P.C. Salmond. "Biotechnological exploitation of bateriophage research." Trends in Biotechnology. 25.1 (2007): 7-15. Web. 4 Apr. 2013. $<$ http://www.sciencedirect.com/science/article/pii/S0167779906002873>.

2) Stella, E. J., De La Iglesia, A. I. \& Morbidoni, H. R. (2009). Mycobacteriophages as versatile tools for genetic manipulation of mycobacteria and development of simple methods for diagnosis of mycobacterial diseases. Revista Argentina de Microbiología, Retrieved from http://www.scielo.org.ar/pdf/ram/v41n1/v41n1a10.pdf

3) Sulakvelidze, Alexander, Zemphira Alavidze, and J.G. Morris. "Bacteriophage Therapy." Antimicrobial Agents and Chemotherapy. 45.3 (2001): 649-659. Print.

$<$ http://www.ncbi.nlm.nih.gov/pmc/articles/PMC90351/>.

4) SEA-PHAGES Laboratory Manual. Chevy Chase, Maryland: Howard Hughes Medical Institute. Retrieved from http://www.hhmi.org/seawiki/download/attachments/14459653/SEA LM In Situ Section Sep 2012.pdf?version $=1$

5) Bertani, G. "Studies on lysogenesis." Journal of Bacteriology. 62.3 (1951): 293-300. Print. $<$ http://www.ncbi.nlm.nih.gov/pmc/articles/PMC386127/pdf/jbacter00613-0069.pdf>.

6) Russell, D. (2012). Phages $d b$. Retrieved from http://phagesdb.org/compare/ 
Table 1. Shown right is an estimation of genome size based on ClaI. ImageJ was used to approximate relative distance.

\begin{tabular}{|r|r|}
\hline \multicolumn{1}{|c|}{ ClaI } \\
\begin{tabular}{|r|r|}
\hline Distance & bp \\
\hline 107.91 & 11500 \\
\hline 142.019 & 6200 \\
\hline 151.32 & 4950 \\
\hline 169.724 & 3800 \\
\hline 177.367 & 3500 \\
\hline 198.461 & 2700 \\
\hline 208.376 & 2500 \\
\hline 246.825 & 1700 \\
\hline 254.888 & 1550 \\
\hline 261.089 & 1450 \\
\hline 269.151 & 1300 \\
\hline 277.834 & 1200 \\
\hline 315.048 & 840 \\
\hline Total & 43190 \\
\hline
\end{tabular}
\end{tabular}


Figure 1. Shown left is an example of a web plate of CharlieBrwn. The plaques cover the plate without completely destroying all of the bacteria, creating a webbed pattern in between plaques.

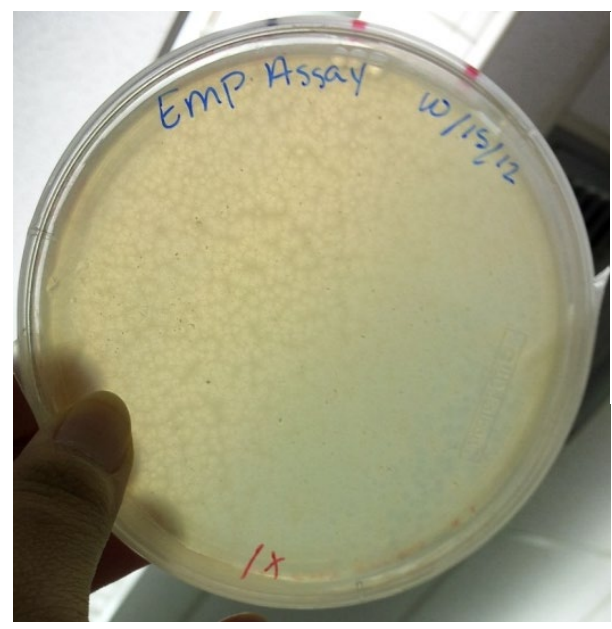


Figure 2. Pictures of completely isolated plaques of CharlieBrwn. A spot test (left) and a web plate (right).

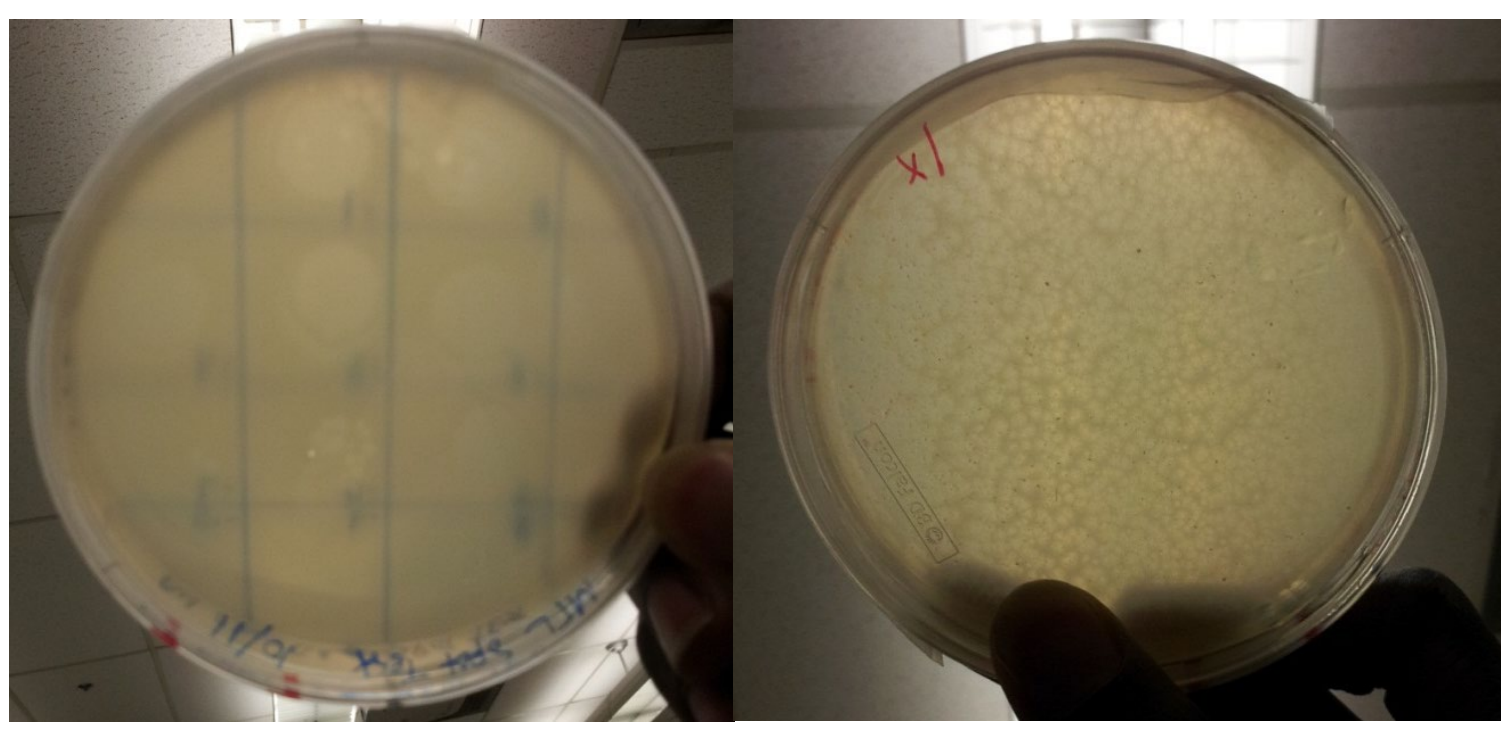


Figure 3. Shown left is the gel electrophoresis of CharlieBrwn. The bands all seem a little faint because of the small amount of DNA.

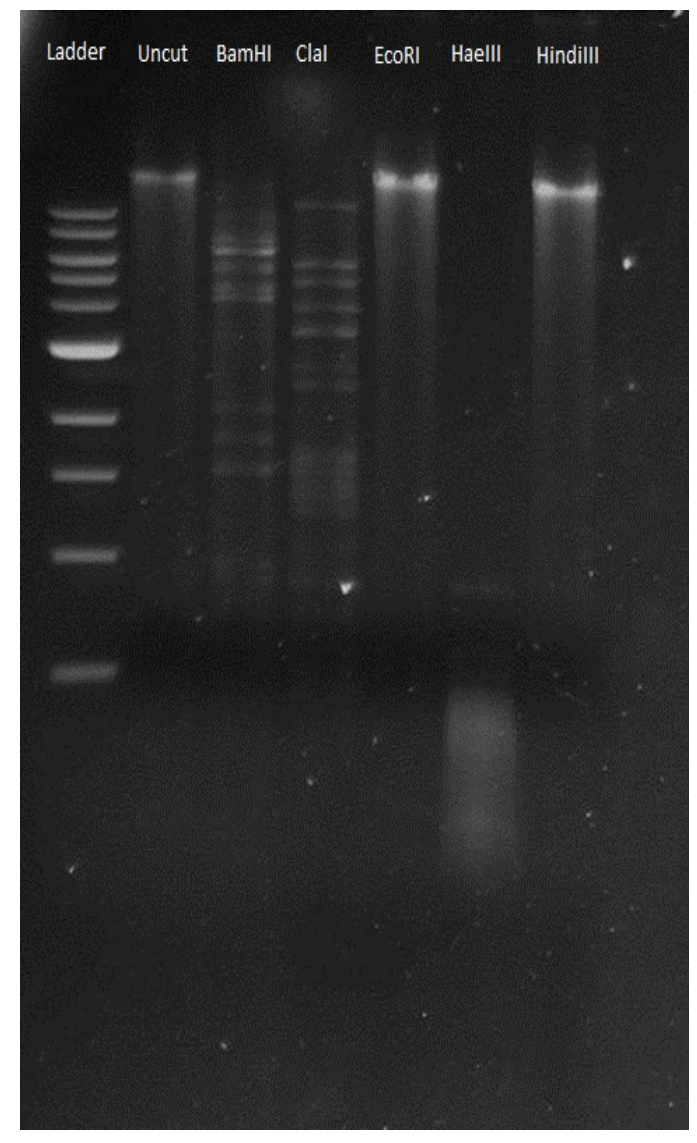


Figure 4. Shown left is an estimation of genome size based on ClaI. The image shows which bands where used in the estimate.

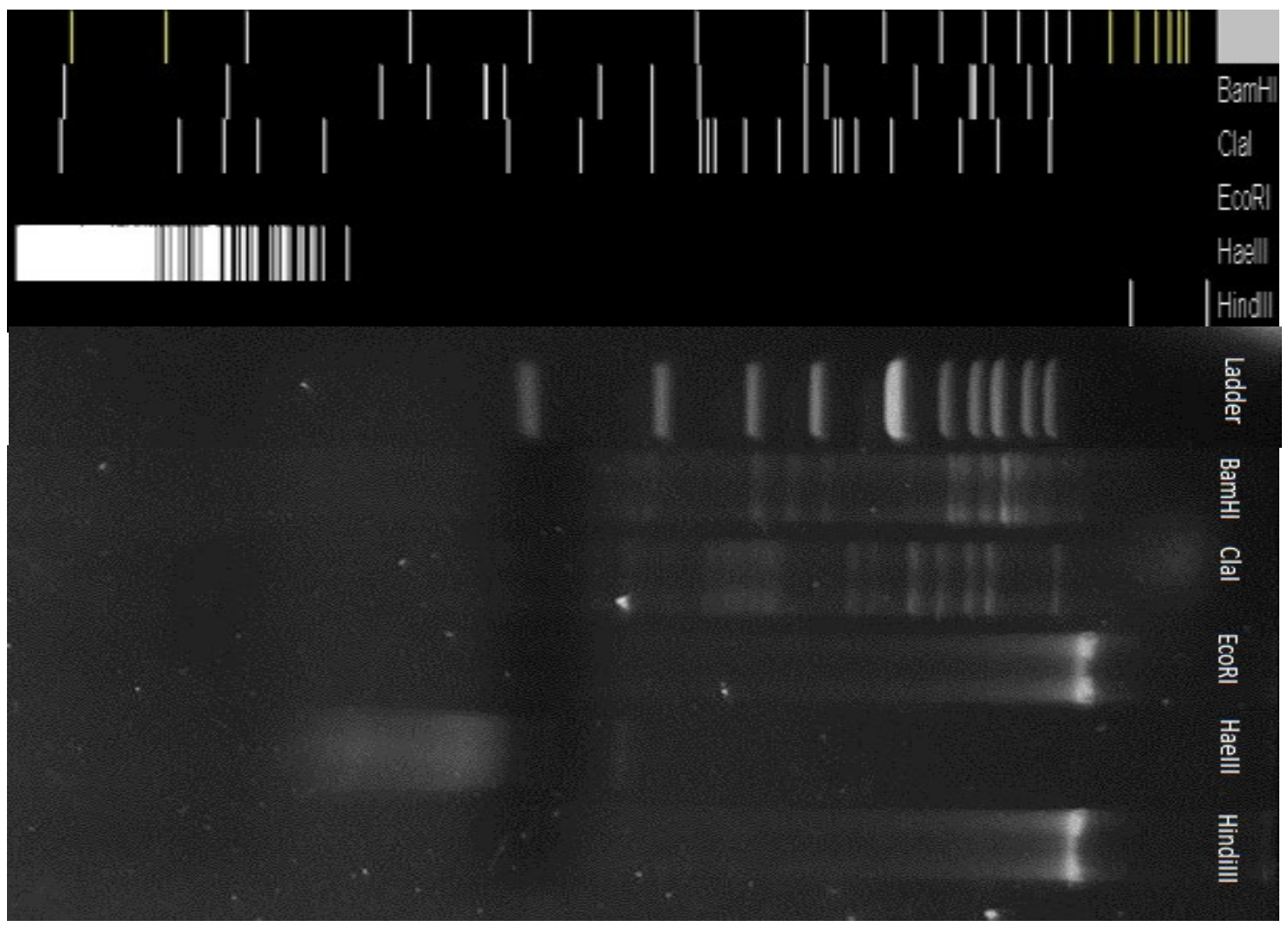


Figure 5. Shown above is the electron micrograph of CharlieBrwn.

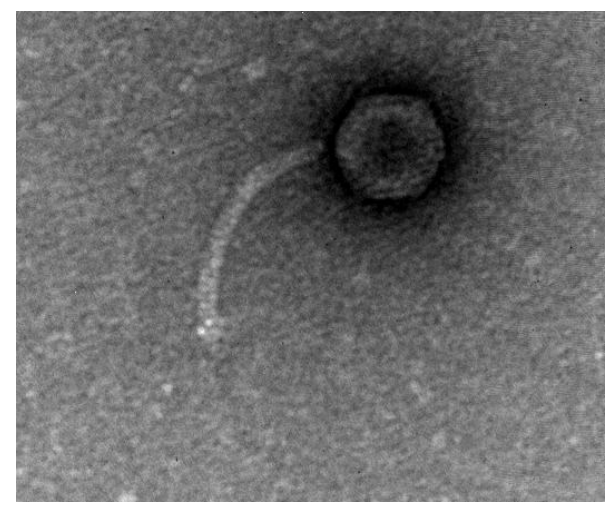


Figure 6. Shown left is the comparison of a virtual digest of a phage from the A2 subcluster (Pukovnik) on top and the gel from CharlieBrwn on bottom.

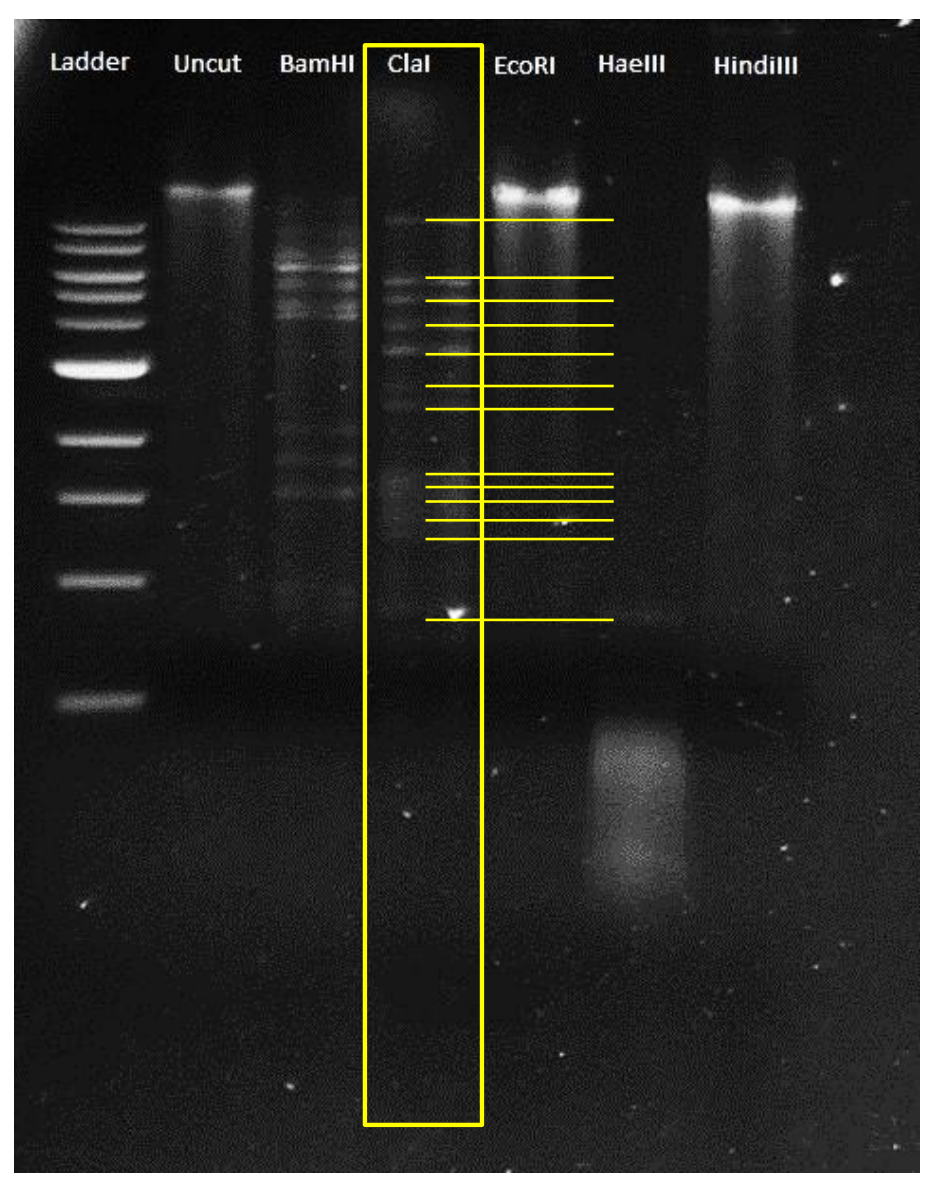


Figure 7. Shown left is the comparison of the electron micrographs of multiple A2 phages (as labeled) to CharlieBrwn (bottom right).
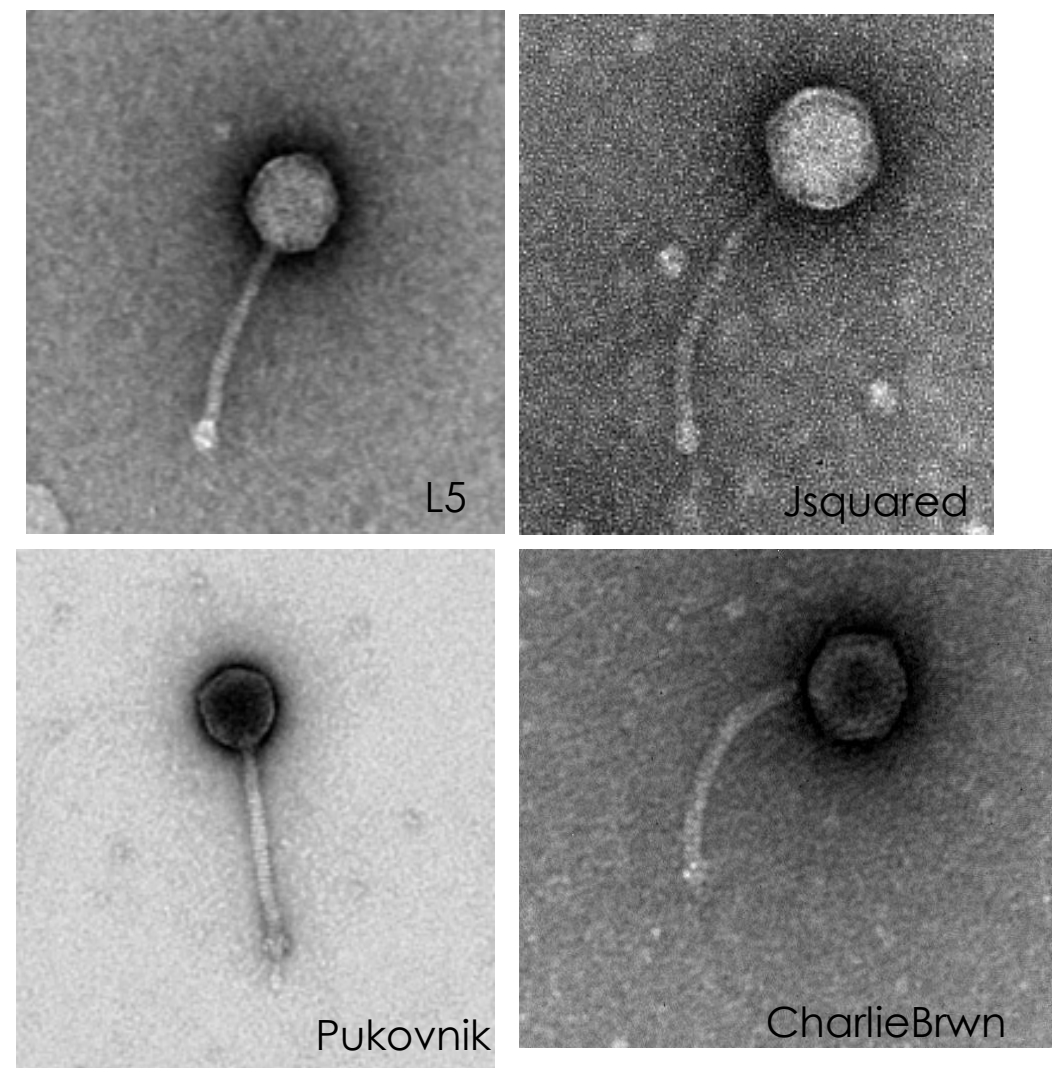